Instabilities in a non-transferred direct current plasma torch operated at reduced pressure

This article has been downloaded from IOPscience. Please scroll down to see the full text article.

2010 J. Phys. D: Appl. Phys. 43085202

(http://iopscience.iop.org/0022-3727/43/8/085202)

View the table of contents for this issue, or go to the journal homepage for more

Download details:

IP Address: 159.226.231.157

The article was downloaded on 07/08/2010 at 12:57

Please note that terms and conditions apply. 


\title{
Instabilities in a non-transferred direct current plasma torch operated at reduced pressure
}

\author{
Heji Huang, Wenxia Pan, Zhiying Guo and Chengkang Wu \\ Institute of Mechanics, Chinese Academy of Sciences, Beijing 100190, People's Republic of China
}

Received 26 September 2009, in final form 20 December 2009

Published 12 February 2010

Online at stacks.iop.org/JPhysD/43/085202

\begin{abstract}
Using an oscilloscope, a high-speed video camera and a double-electrostatic probe system, the periodicity and amplitude of the fluctuations in arc voltage, jet luminance and ion saturation current of a plasma jet were monitored to investigate various sources of instabilities and their effects in a non-transferred dc plasma torch operated at reduced pressure. The results show that besides a $300 \mathrm{~Hz}$ main fluctuation inherited from the power supply, arc voltage fluctuation of $3-4 \mathrm{kHz}$ with an amplitude less than $5 \%$ of the mean voltage was mainly affected by the total gas flow rate. The arc voltage fluctuation can affect the energy distribution of the plasma jet which is detectable by electrostatic probes and a high-speed video camera. The steadiness of energy transfer is also affected by the laminar or turbulent flow state of the plasma.
\end{abstract}

\section{Introduction}

Coating is one of the most important applications of thermal plasma materials processing, and is a rapidly growing area of research and industry. In a typical thermal plasma coating process, the plasma is usually used as the momentum and enthalpy source. Feedstock material is heated and accelerated in the plasma flow and then directed to a target surface of the workpiece to form the desired coating. Although new thermal plasma sources are being developed continuously, non-transferred direct current (dc) plasma, owing to its versatility and high cost efficiency, still have the largest share in thermal plasma coating industry [1]. Coatings deposited by dc plasma have been applied in a broad variety of industrial applications, such as in aeronautics, auto industry, environmental engineering and medical industries [2]. However, market with increasing demanding for different coatings of high-end applications requires better consistency products. This calls for the specific management of every aspect of processing, including the improvement of thermal plasma equipment, the achievement of more consistent feedstock materials, pretreatment of the sample surface, on-line process control, post-processing and the standardization of testing. All the dispersions in these processes should be addressed and controlled for reduced variability. Among them, the plasma flow is at the first stage of a coating deposition process, and hence the characteristics of which play an important role in the coating quality.
Unfortunately, the behaviour of a thermal dc plasma flow is not static but rather dynamic. Interactions among electromagnetic, fluid dynamic and thermal fields inside the dc plasma torch result in non-linear time-dependent instabilities and distributions of the associated plasma quantities. To improve the consistency of the coating, it is essential to identify the origins of such fluctuations before controlling them.

Intensive studies have been carried out both experimentally and theoretically regarding the fluctuation behaviour of dc plasma torches. Since most of the power supplies for dc plasma coating purpose are nearly constant-current sources, time-dependent arc voltage spectrum is widely accepted as an informative method to describe the dynamic energy fluctuation of a dc plasma torch. Till now, it is found that an erratic arc voltage evolution mainly corresponds to the movement of the arc root, which arises out of the time-dependent balance of electromagnetic body force and gas dynamic drag force affecting the current path from the anode to the cathode $[3,4]$. These forces are controlled by the factors of torch geometry, plasma gas type and flow rate, input current and other plasma generating parameters. Recently, Helmholtz oscillation arising from torch channels has also been suggested to be another reason for fine structures in arc voltage spectra $[5,6]$.

Variation of arc voltage does change the enthalpy of the plasma jet. In some experiments, it is confirmed that the change in temperature and velocity of the injected powders in the plasma follow the arc voltage fluctuations [7,8]. However, 
even if no arc voltage change exists, gradients of temperature and velocity in the plasma jet will not vanish because of the cooling condition and turbulence of the plasma flow. Since feedstock materials are usually injected into the plasma jet region instead of the arc region, the flow condition of the plasma jet also affects the trajectory and the heating history of the injected feedstock materials remarkably.

From these points of view, in addition to controlling the arc voltage fluctuation, it is also important to control the stability of the plasma jet flow to achieve a highly reliable and reproducible process. In this research, a non-transferred dc plasma torch with an abruptly expanded anode and a set of inter-electrode inserts was designed to generate a plasma of relatively large volume with moderate parameter gradients at reduced pressure. The torch design is similar to that reported in $[9,10]$. Between the cathode and the anode of the non-transferred dc plasma torch, an insert of floating electric potential, with a relatively long, narrow channel through which the arc column passes, was designed to generate the plasma under almost a fixed arc length condition. That is, with the special torch structure, the arc can only find its suitable attachment position on the anode surface within an almost fixed and narrow region under a given gas feeding condition and at a given arc current. In this kind of torch, the change in arc column length caused by the movement of the arc root on the anode surface could generally be neglected compared with its long column passing through the inter-electrode insert. Thus, the fluctuation component of arc voltage originating from the length change of the arc column can be kept generally at a negligible level [11]. Experimental results indicate that this kind of torch can generate a plasma jet of laminar or turbulent flow state at reduced pressure by regulating the gas flow rate and chamber pressure [12]. The obtained laminar plasma jet can be used for surface modification and coating with significantly improved process controllability compared with a conventional turbulent plasma jet. The object of this paper is to characterize the instabilities in pure argon plasma generated by this torch at reduced pressure through approaches of acoustic analysis, arc voltage analysis, probe measurement and optical observation.

\section{Experimental details and analysis methods}

Figure 1 shows the schematic diagram of the experimental system. An arc goes from the tip of the cathode, through the long channel of the inter-electrode insert and reaches the upper surface of an abruptly expanded anode. The size of the channel varies from 7 to $12 \mathrm{~mm}$ while that of the anode ranges from 50 to $80 \mathrm{~mm}$ in diameter. All the electrodes are water-cooled. The acoustic property of the plasma torch was characterized using a digital recorder before generating the plasma to evaluate the geometric effect on Helmholtz oscillation. The equation

$$
f=\frac{a}{2 \pi} \sqrt{\frac{A}{V L}},
$$

deduced from the vibration of small mass of gas in the neck with section area $A$ and length $L$ of a cavity with volume $V$, can be used to describe the frequency of Helmholtz oscillation in such a system without considering the viscous effects. In

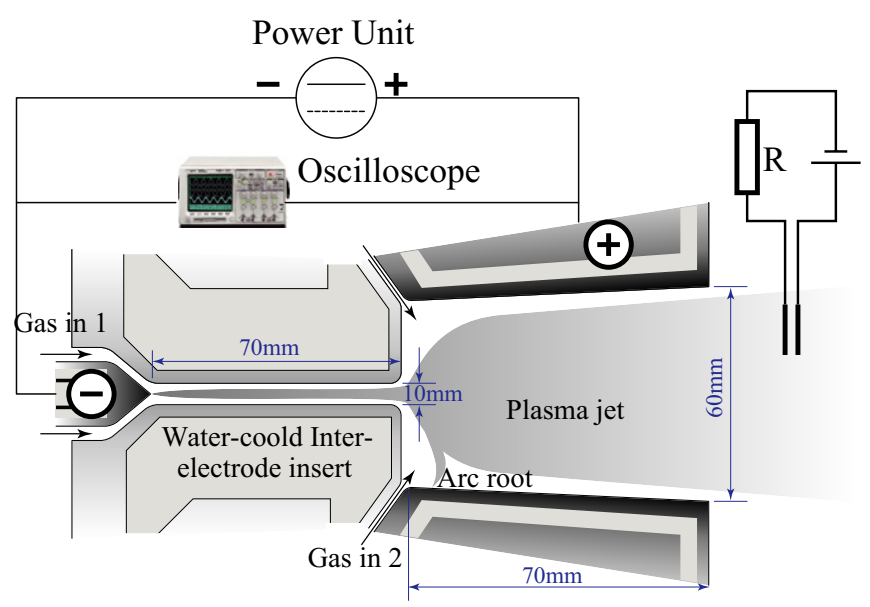

Figure 1. Schematic diagram of the experimental system. (This figure is in colour only in the electronic version)

the equation, $a$ stands for the speed of sound and $f$ stands for the Helmholtz oscillation frequency. The terms in the square root are just determined by the geometry of the torch and thus a shape factor $K=1 / 2 \pi \sqrt{A / V L}$ is defined. However, in a real plasma torch, since the fine structure is rather complicated, this shape factor is not easy to be simply calculated. By recording and analysing sound waves for different argon gas feeding rates under atmospheric condition in cold state, the acoustic frequencies of the torch were confirmed. Since the speed of sound for pure argon at room temperature is about $315 \mathrm{~m} \mathrm{~s}^{-1}$, it was possible to determine the shape factor $K$ for the specific torch.

Argon was used as the plasma gas and the gas flow rates varied from 4.2 to $21 \mathrm{slm}$. The chamber pressure was maintained between 0.5 and $10 \mathrm{kPa}$ and the arc current between 80 and $100 \mathrm{~A}$. When the plasma was generated, a doubleelectrostatic probe system was adopted to monitor the ion saturation current in the plasma plume near the centre of the torch exit. The double-electrostatic probe system consists of an $18 \mathrm{~V}$ bias voltage supply, a $500 \Omega$ resistance and two tungsten electrodes of $0.3 \mathrm{~mm}$ diameter. An oscilloscope with nanosecond time resolution was used to monitor the dynamic voltage change on the ends of the resistance. Previous studies have shown that under the $18 \mathrm{~V}$ bias voltages, the obtained signal represents the ion saturation current in the plasma plume under the typical studied conditions. Another oscilloscope was used to monitor the arc voltage and the current spectra simultaneously. A high-speed video camera capable of $1.2 \times 10^{5}$ frames per second (fps) was used to obtain the instantaneous plasma jet images through the chamber window, with a recording speed of $2500 \mathrm{fps}$ and an exposure time of $1 \mu \mathrm{s}$ per frame.

\section{Results and discussion}

When cold Ar was fed only through the cathode cavity (marked in figure 1 as Gas in 1, hereafter represented by inlet 1) into the torch, the resulting sound waveforms recorded by the digital recorder are shown in figure $2(a)$. When the gas feeding rate is as low as $2.8 \mathrm{slm}$, the recorded waveform is relatively 
Ar feeding only through inlet 1
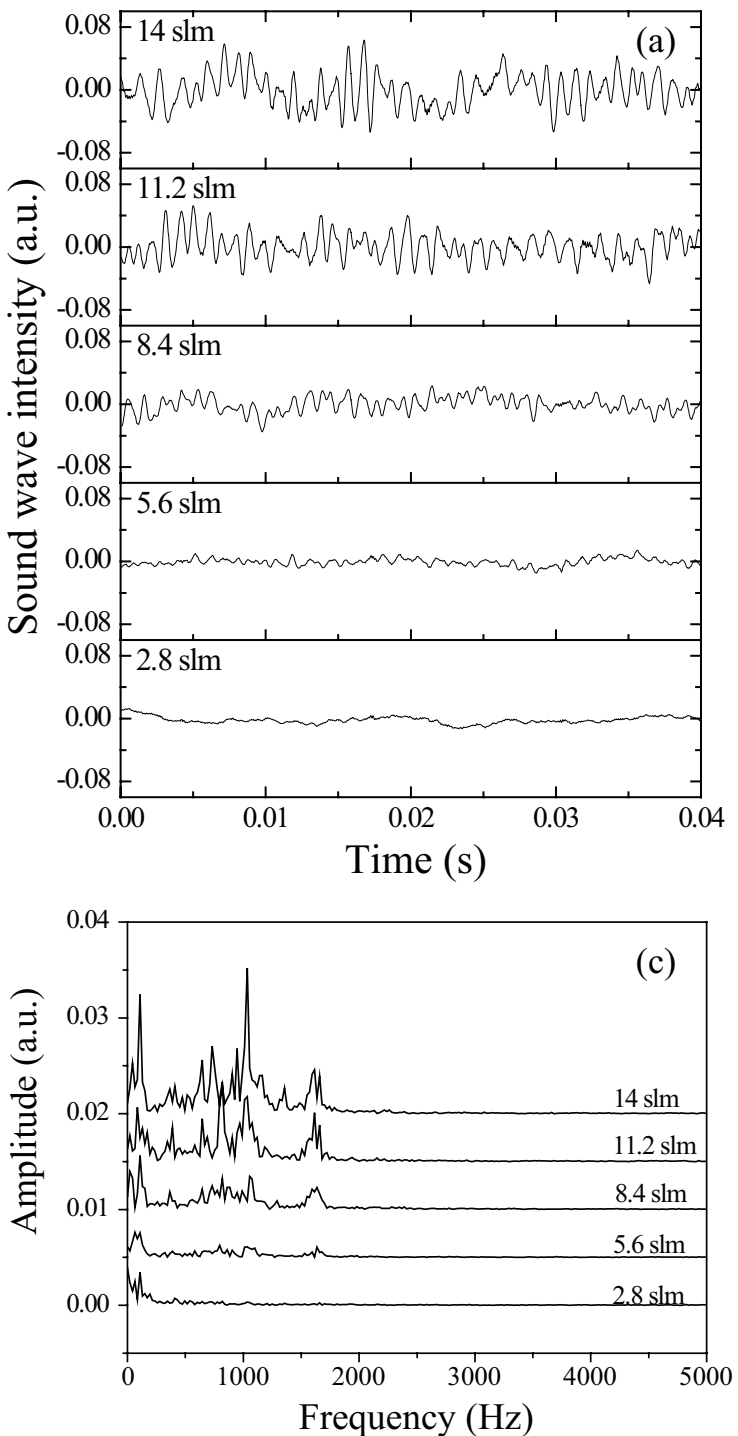

Ar feeding through both inlets 1 and 2
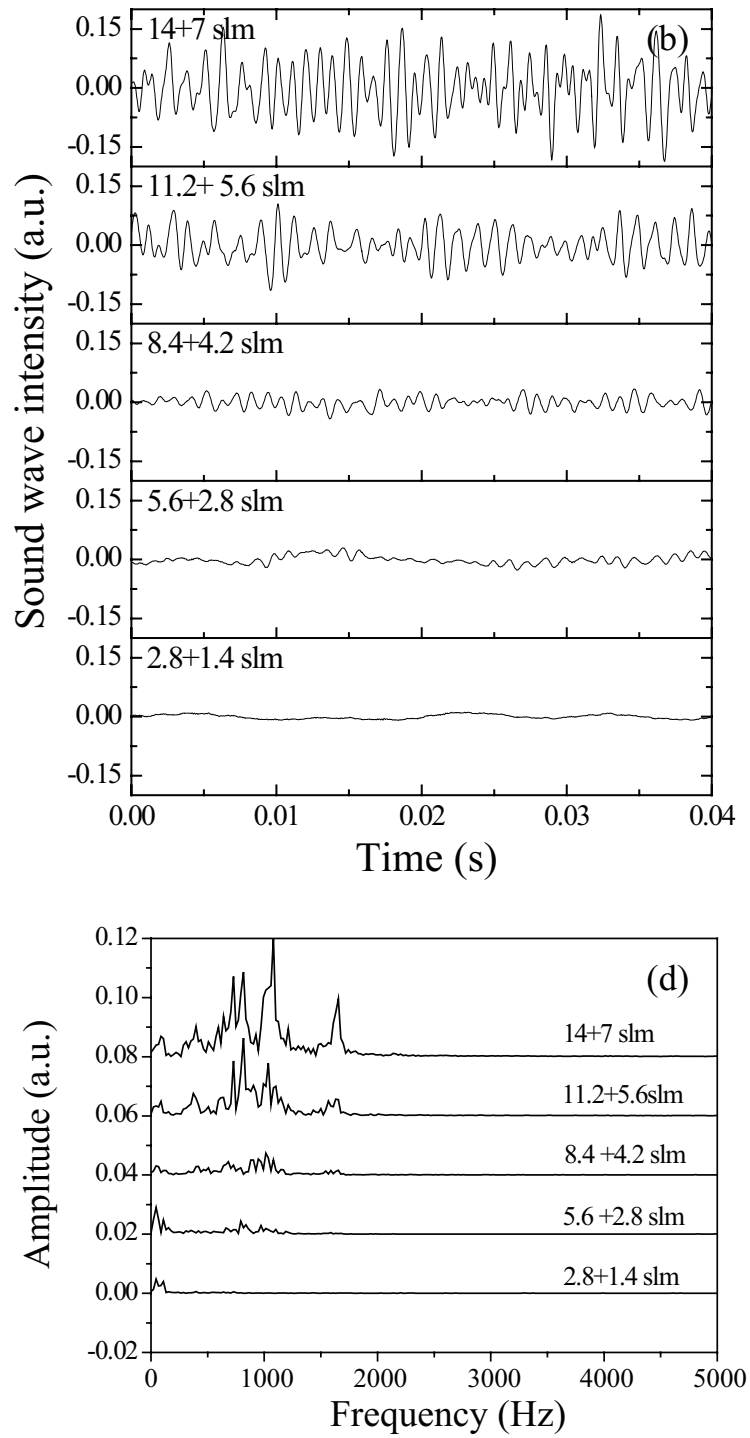

Figure 2. Cold state acoustic properties of the torch with various gas feeding conditions under atmospheric condition. (a) Sound wave spectra of the torch when Ar is fed through inlet 1 only; $(b)$ sound wave spectra of the torch when Ar is fed through both inlets 1 and 2; $(c)$ frequency analysis of the spectra in $(a) ;(d)$ frequency analysis of the spectra in $(b)$.

smooth. With the increase in the gas feeding rate, notable higher frequency sound waves appear. Since this is in the cold state and no plasma exists, such sound waves originate only from Helmholtz oscillation.

In the case of plasma generation, gas is fed into the torch not only through inlet 1 but also through inlet 2 (marked in figure 1 as Gas in 2). However, in the cold state, additional feeding of gas through inlet 2 has negligible effect on the sound waveforms recorded. Figure $2(b)$ shows the result when half amount of the gas fed through inlet 1 is additionally fed into the torch through inlet 2. Similar waveforms compared with figure 2(a) are seen when the gas flow rate through inlet 1 is the same. These results show that in the present dc plasma torch, mainly the cathode cavity with the long inter-electrode channel forms the Helmholtz resonator. Frequency analyses of the sound waveforms in figures $2(a)$ and $(b)$ are shown in figures $2(c)$ and $(d)$, respectively. It clearly shows that when the gas flow rate is too low, oscillation cannot be excited. At higher gas flow rates, the amplitude of the oscillation increases while the frequencies of which remain almost the same. The multifrequency oscillation might result from fine structures of the inter-electrode insert channel, which involve different values of $K$ factor. At room temperature, the speed of sound for pure argon is about $315 \mathrm{~m} \mathrm{~s}^{-1}$, thus the shape factor $K$ of the specific torch is in a series of 2.3, 2.6, 3.2 and 5.1 in the unit of $\mathrm{m}^{-1}$ for the main oscillation frequency of $728,816,1012$ and $1615 \mathrm{~Hz}$. These shape factors can be used to estimate Helmholtz oscillation frequencies when the plasma is generated.

When the plasma is generated, in all the cases studied, the arc voltage spectrum is dominated by a $300 \mathrm{~Hz}$ fluctuation, which has been clarified to originate from the tri-phase rectified power supply when the rectification is not well synchronized. The characteristics of the power supply were described in [11]. Such a low frequency fluctuation (hereafter represented by LFF) is independent of any change in the operating parameters. However, higher frequency fluctuations (hereafter 

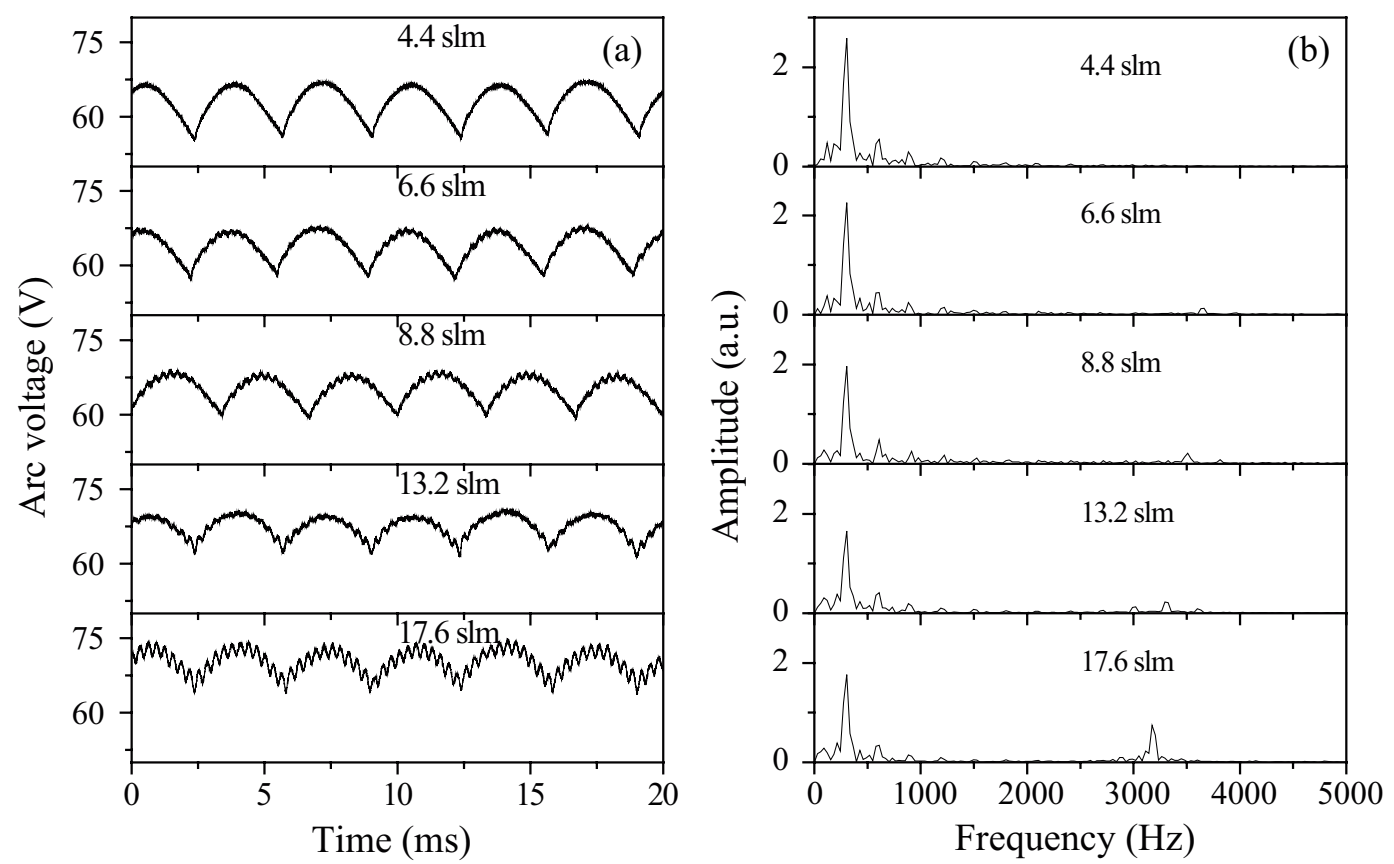

Figure 3. Dependence of the arc voltage fluctuations on total gas flow rate for pure argon plasma at $3000 \mathrm{~Pa}:(a)$ arc voltage spectra; $(b)$ FFT analysis of the frequencies of the arc voltage spectra.

represented by HFF) of the arc voltage are somewhat sensitive to the plasma generating conditions. From figure 3(a), a clear dependence of HFF on the total gas flow rate can be seen: when the chamber pressure was kept constant at $3 \mathrm{kPa}$, no HFF was observed at a gas flow rate of $4.4 \mathrm{slm}$, but at elevated gas flow rates, the superposing of HFF and LFF in the voltage spectrum becomes noticeable. At higher gas flow rates, the HFF become more pronounced. However, both the absolute value of $\mathrm{HFF}$ $(\Delta V)$ and the relative change in voltage $(\Delta V / V$, where $V$ is the mean voltage) are small. The largest fluctuation is smaller than $5 \%$ at a gas flow rate of $17.6 \mathrm{slm}$.

Using fast Fourier transformation (FFT) analysis method, the frequencies of the arc voltage spectra were analysed. Figure $3(b)$ shows that the arc voltage spectrum is quite clean. Unlike that of conventional dc plasma torches, the arc voltage spectrum of the specially designed torch operated under the conditions described above comprises only a few explicit frequencies, most of which are the $300 \mathrm{~Hz}$ LFF and its harmonics. With the increase in the plasma gas flow rate from 6.6 to $17.6 \mathrm{slm}$, the frequency of $\operatorname{HFF}\left(F_{\mathrm{H}}\right)$ decreases from 3.7 to $3.2 \mathrm{kHz}$. Previous studies have shown that under such plasma generation conditions, pure argon plasma has a diffused type arc root attachment [13]. Direct relationship between the arc root behaviour and the arc voltage spectra cannot be established. Thus, such HFF are likely to originate from Helmholtz oscillation described above.

When plasma is generated, equation (1) is also applicable to the estimation of Helmholtz oscillation frequency. However, the speed of sound in the cathode cavity and the inter-electrode channel are not easily measured. It is essential to estimate the plasma density through the measurement of specific enthalpy of the plasma in the inter-electrode channel. Thus equation (1) can be revised to $f=K \sqrt{\gamma_{\mathrm{c}}(\gamma-1 / \gamma)\left(P_{\mathrm{c}} / P\right) h}$ [14]. Here, subscript $c$ represents the quantity of the cathode cavity.

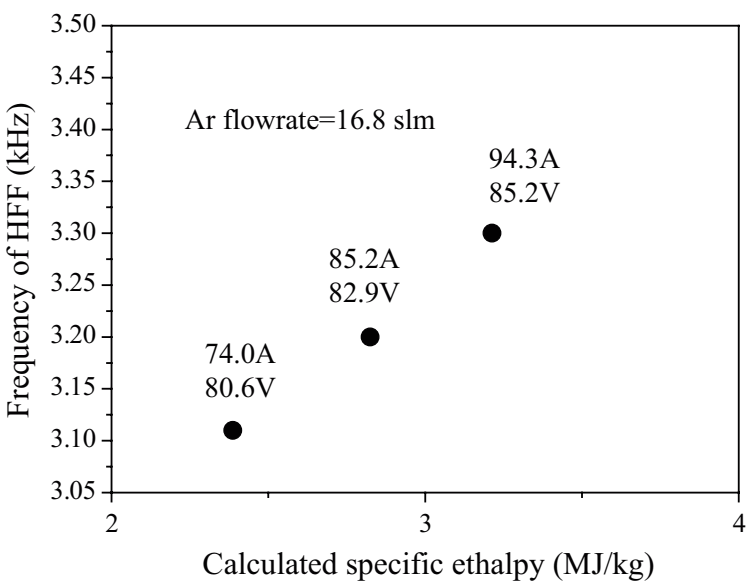

Figure 4. Dependence of measured $F_{\mathrm{H}}$ on calculated specific enthalpy.

$P$ and $h$ stand for the pressure and specific enthalpy in the inter-electrode channel, respectively. From this equation, it is seen that the oscillation frequency is almost proportional to the specific enthalpy of the plasma when the pressure ratio $P_{\mathrm{c}} / P$ does not change much. The dependence of measured $F_{\mathrm{H}}$ on calculated specific enthalpy (assuming the thermal efficiency of the torch to be 20\%) is plotted in figure $4 . K$ is then calculated to be in the range of $2.5-3$, which is in similar range as that obtained from figure $2(d)$.

Generally, fluctuations in the plasma jet result from the combination of energy and flow perturbations. By using an electrostatic probe system, dynamic ion saturation current fluctuations of the plasma jet at the torch exit are shown in figure 5. The detected spectrum of ion saturation current can be used to describe the fluctuation behaviour of the plasma jet. In figure 5 , the chamber pressure is $10 \mathrm{kPa}$, and the total gas flow rate of argon changes from 8.4 to $21 \mathrm{slm}$. It can be 
seen that when the gas flow rate is small in the case of 8.4 and $12.6 \mathrm{slm}$, the superposition of higher frequency perturbations on the intrinsic $300 \mathrm{~Hz}$ fluctuation is clear, which indicates that the fluctuation of ion saturation current is mainly inherited from the arc voltage fluctuation and there is no remarkable flow perturbations in these cases. However, when the total gas flow rate is $21 \mathrm{slm}$, the ion saturation current signal is disordered, and no obvious periodicity can be seen. In this situation, the plasma jet is in a turbulent state where flow perturbations in the plasma torch mess the energy distributions of the plasma jet. As a result, periodical fluctuation characteristics of the arc voltage can hardly be detected in the ion saturation current spectrum. When the gas flow rate is $16.8 \mathrm{slm}$, a transition between the two modes is observed.

With the increase in the chamber pressure, the dynamic stability of the plasma jet also becomes worse. Figure 6 compares the ion saturation current signals at different chamber pressures of $0.5,5$ and $10 \mathrm{kPa}$. The arc current is kept at $80 \mathrm{~A}$ and the total gas flow rate is $16.8 \mathrm{slm}$. From figure $6(a)$, it can be clearly seen that the detected ion saturation current signal becomes more disordered at higher chamber pressures.

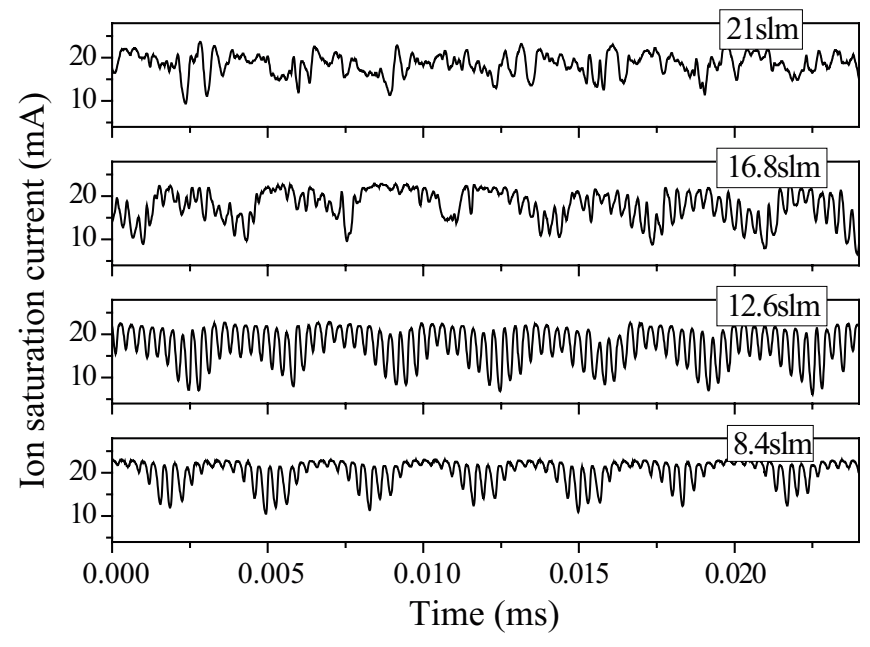

Figure 5. Dependence of ion saturation current on gas flow rate with a chamber pressure of $10 \mathrm{kPa}$ and an arc current of $80 \mathrm{~A}$.

(a)

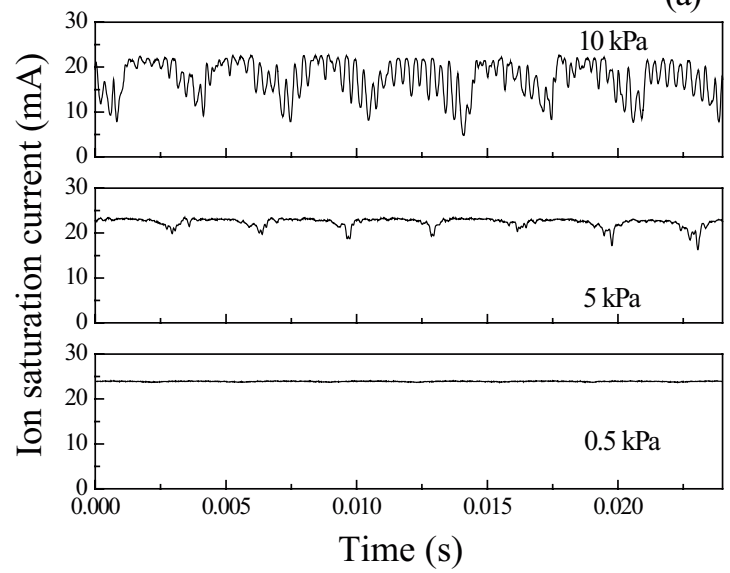

Larger perturbations with a higher frequency are superposed on the $300 \mathrm{~Hz}$ intrinsic fluctuations when the chamber pressure is increased to $10 \mathrm{kPa}$. It is also interesting to see that when the chamber pressure is low, in particular for the case of $0.5 \mathrm{kPa}$, the $300 \mathrm{~Hz}$ fluctuation is suppressed in the plasma jet. A possible reason is that at a lower chamber pressure, the plasma jet flow is more effectively homogenized in the abruptly expanded anode. Figure $6(b)$ shows the possibility of ion saturation current distributions at different chamber pressures. It is seen that with the increase in chamber pressure, wider distribution of ion saturation current is observable, together with a lower average ion saturation current level. This result shows that at elevated pressures, the electric conductivity of the plasma decreases owing to more intensive collisions between electrons and ions.

A previous study showed that gas flow rate and chamber pressure significantly affect the laminar or turbulent state of flow of the plasma [12]. Turbulent flow develops at high gas flow rates and chamber pressures. The results in figures 5 and 6 suggest that turbulence in the plasma jet can further amplify the time-dependent instabilities of the associated plasma parameters.

Figure 7(a) shows the photo of the obtained plasma jet taken by a high-speed video camera when the chamber pressure is up to $10 \mathrm{kPa}$ with a total gas flow rate of $16.8 \mathrm{slm}$. The camera speed was set to be $2500 \mathrm{fps}$ and the exposure time to be $1 \mu \mathrm{s}$. A previous study showed that when the plasma jet is in a turbulent state, twisted plume can be clearly seen when the exposure time is as short as $10 \mu \mathrm{s}$. However, in this study, even when the exposure time is 10 times shorter, no twist and distortion were observed, showing that the plasma jet is mainly in a laminar flow state. However, there are observable changes in brightness and shape of the plasma jet if a series of images are compared. Figures 7(a)-(e) show a consequent series of photos with intervals of 5 frames ( $2 \mathrm{~ms}$ in time). A periodical change in the width and brightness of the plasma jet can be seen. By analysing 200 images taken in $80 \mathrm{~ms}$, the periodicity of the plasma jet intensity change is confirmed to be $300 \mathrm{~Hz}$, which corresponds to the arc voltage fluctuations originating from the rectified power supply.

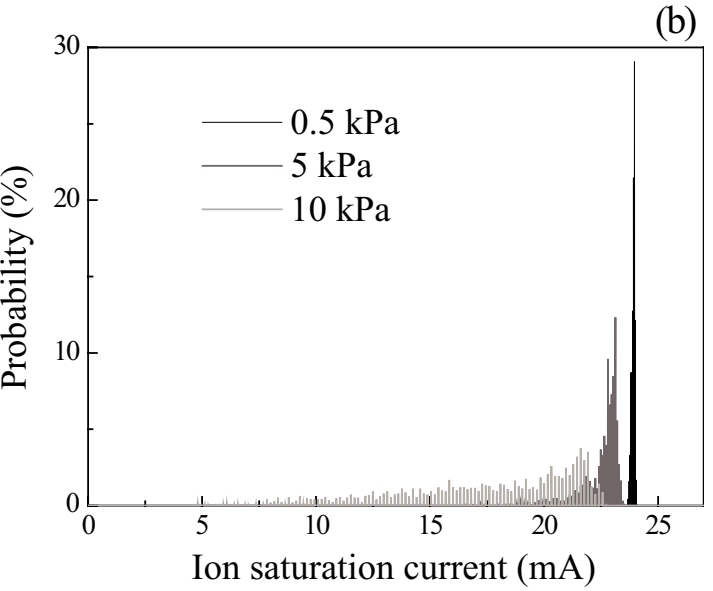

Figure 6. Effect of chamber pressure on the measured axial ion saturation current signals. (a) Time dependent ion saturation current spectra; $(b)$ distribution of ion saturation current. 

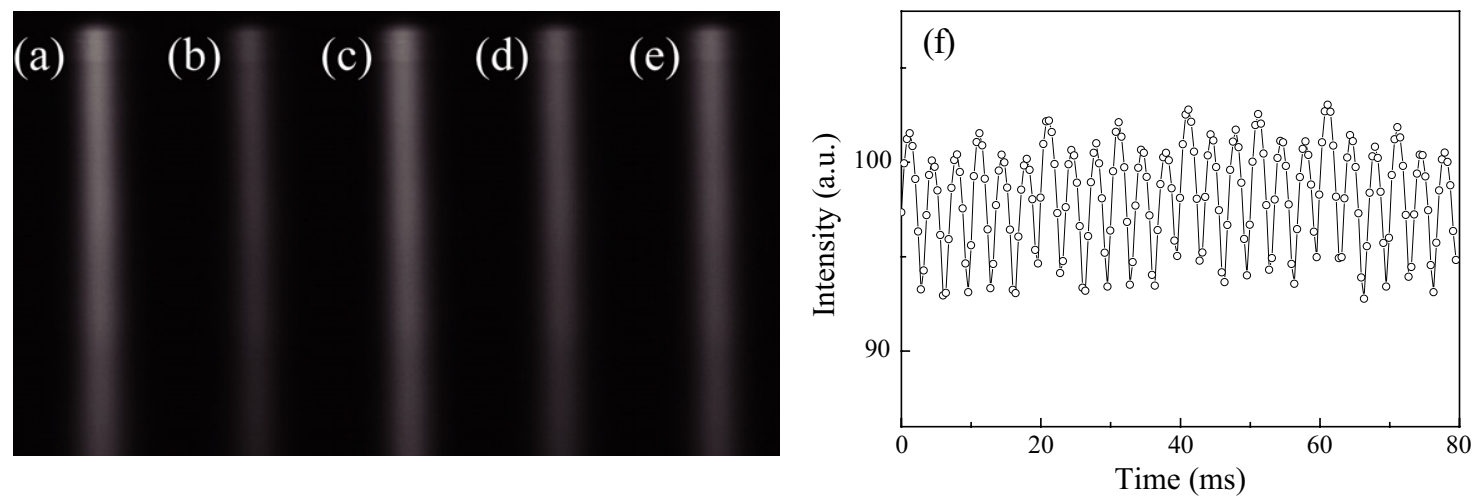

Figure 7. Time-dependent stability of the plasma jet generated at $80 \mathrm{~A}, 10 \mathrm{kPa}$ with a total gas flow rate of $16.8 \mathrm{slm}$. $(a)-(e)$ High-speed video camera photos of the plasma jets with time intervals of $2 \mathrm{~ms}$ and exposure time of $1 \mu \mathrm{s} ;(f)$ variation of plasma jet intensities with time.

\section{Conclusions}

An experimental study on the instabilities in a non-transferred dc plasma torch operated at reduced pressure shows that a quite clean arc voltage spectrum consisting of the superposing of a few explicit frequencies can be obtained. A $300 \mathrm{~Hz}$ fluctuation and its harmonics inherited from the torch power supply dominate. Higher frequency fluctuations of arc voltage exist under some conditions, and the amplitude of the high frequency fluctuation is smaller than 5\%. Such a high frequency fluctuation possibly arises from Helmholtz oscillation because of the specific torch geometry. In spite of $8 \%$ fluctuation amplitude of the low frequency perturbation resulting from the power supply, laminar plasma flow can be maintained under optimized plasma generation conditions.

Dynamic measurement of the ion saturation current is a fast response method to reveal the energy fluctuations in a plasma jet generated at reduced pressures of $0.5-10 \mathrm{kPa}$. The instabilities in the plasma jet are affected by the combination of fluctuations from both arc voltage and flow state. With laminar plasma flow, the effects of arc voltage fluctuation can be suppressed in the plasma jet, particularly at lower chamber pressures. On the other hand, flow turbulence promotes the time-dependent instabilities in the plasma jet. In order to achieve a highly consistent coating, controlling of both arc voltage and plasma flow instabilities is important.

\section{Acknowledgment}

This work is supported by the National Natural Science Foundation of China under Grant Nos 50836007, 50702064 and 10921062 .

\section{References}

[1] Fauchais P et al 2006 Developments in direct current plasma spraying Surf. Coat. Technol. 201 1908-21

[2] Boulos M I, Fauchais P and Pfender E 1994 Thermal Plasmas (New York: Plenum) p 452

[3] Yang G and Heberlein J 2007 Anode attachment modes and their formation in a high intensity argon arc Plasma Sources Sci. Technol. 16 529-42

[4] Ghorui S et al 2007 Probing instabilities in arc plasma devices using binary gas mixtures Phys. Rev. E 76016404

[5] Delair L et al 2005 Helmholtz behavior of a nitrogen plasma arc chamber High Temp. Mater. Process. $9583-97$

[6] Coudert J F and Rat V 2008 Influence of configuration and operating conditions on the electric arc instabilities of a plasma spray torch: role of acoustic resonance J. Phys. D: Appl. Phys. 41205208

[7] Bisson J F, Gauthier B and Moreau C 2003 Effect of plasma fluctuations on in-flight particle parameters J. Thermal Spray Technol. 12 38-43

[8] Moreau C et al 2005 Diagnostics for advanced materials processing by plasma spraying Pure Appl. Chem. 77443

[9] Pan W X et al 2006 Experimental study on the thermal argon plasma generation and jet length change characteristics at atmospheric pressure Plasma Chem. Plasma Process. $26335-45$

[10] Pan W X et al 2001 Generation of long, laminar plasma jets at atmospheric pressure and effects of flow turbulence Plasma Chem. Plasma Process. 21 23-35

[11] Pan W X, Meng X and Wu C K 2006 Arc voltage fluctuation in DC Laminar and turbulent plasma jets generation Plasma Sci. Technol. 8 416-21

[12] Huang H J et al 2008 Laminar/turbulent plasma jets generated at reduced pressure IEEE Trans. Plasma Sci. 36 1052-3

[13] Huang H J et al 2008 Arc root motion in an argon-hydrogen dc plasma torch IEEE Trans. Plasma Sci. 36 1050-1

[14] Coudert J F, Rat V and Rigot D 2007 Influence of Helmholtz oscillations on arc voltage fluctuations in a de plasma spraying torch J. Phys. D: Appl. Phys. 40 7357-66 